\title{
Sex-specific histone modifications in mouse fetal and neonatal germ cells
}

\author{
Yukiko Kawabata1, Asuka Kamio1,2, Yuko Jincho1, Akihiko Sakashita1, Tomoya Takashima1, \\ Hisato Kobayashi², Yasuhisa Matsui ${ }^{3}$ \& Tomohiro Kono*,1 \\ ${ }^{1}$ Department of Bioscience, Tokyo University of Agriculture, Setagaya, Tokyo, Japan \\ ${ }^{2}$ NODAI Genome Research Centre, Tokyo University of Agriculture, Setagaya, Tokyo, Japan \\ ${ }^{3}$ Cell Resource Centre for Biomedical Research, Institute of Development, Aging \& Cancer, Tohoku University, Sendai, Miyagi, Japan \\ *Author for correspondence: tomohiro@nodai.ac.jp
}

\begin{abstract}
Aims: Epigenetic signatures of germline cells are dynamically reprogrammed to induce appropriate differentiation, development and sex specification. We investigated sex-specific epigenetic changes in mouse fetal germ cells (FGCs) and neonatal germ cells. Materials \& methods: Six histone marks in mouse E13.5 FGCs and P1 neonatal germ cells were analyzed by chromatin immunoprecipitation and sequencing. These datasets were compared with transposase-accessible chromatin sites, DNA methylation and transcriptome. Results: Different patterns of each histone mark were detected in female and male FGCs, and H3K4me3/H3K27me3 bivalent marks were enriched in different chromosomal regions of female and male FGCs. Conclusion: Our results suggest that histone modifications may affect FGC gene expression following DNA methylation erasure, contributing to the differentiation into female and male germ cells.
\end{abstract}

First draft submitted: 7 November 2018; Accepted for publication: 2 January 2019; Published online: 22 January 2019

Keywords: ATAC-seq $\bullet$ DNA methylation $\bullet$ epigenetics $\bullet$ fetal germ cells $\bullet$ gene expression $\bullet$ histone modifications - sex differences $\bullet$ transcriptome

Epigenomic signatures regulate gene expression and are transmitted to the next generation through the germline. Epigenetic marks are dynamically reprogrammed during germ cell differentiation to generate female and male gametes with unique epigenomic information. In mice, primodial germ cells (PGCs) - which are a repository for epigenomic memory - are specified as B lymphocyte-induced maturation protein (Blimp) 1- and PR/SET domain (Prdm) 14-positive precursors in the most proximal epiblast at 6.25 days post coitus (dpc) [1,2]. Blimp1 and Prdm 14 maintain PGCs in a pluripotent state that distinguishes them from somatic cells. Sex differentiation of gonads is induced by transient expression of the sex-determining region Y gene, which is expressed in pre-Sertoli cells of the male genital ridge at $11.5 \mathrm{dpc}$ [3]. PGCs are dimorphic and sexually bipotent as they migrate toward the genital ridge [4].

PGCs undergo global epigenetic reprogramming - including exchange of histone variants, remodeling of histone modifications and erasure of DNA methylation - during migration and proliferation [5-8]. We previously generated single-base genome-wide DNA methylome maps of developing PGCs [9] and adult gametes [10] by post-bisulfite adaptor-tagging and demonstrated that DNA is globally hypomethylated $(<2-3 \%)$ in both female (f) PGCs/fetal germ cells (FGCs) and male (m) FGCs by embryonic day (E) 13.5. After global demethylation, FGCs undergo fate determination; however, while fFGCs maintained their hypomethylated status $(2.3 \%)$ in non-growing stage oocytes in day 1 (P1) pups - with maternal methylation occurring during oocyte growth showing up to $40 \%$ coverage $[10,11]-$ mFGCs acquire a male-specific methylation $(76.1 \%)$ status by birth, which is similar to the sperm methylome $[10,12]$. Interestingly, a transcriptome analysis revealed that $\mathrm{fFGCs}$ and $\mathrm{mFGCs}$ are already distinguishable at $13.5 \mathrm{dpc}$ by their gene expression profiles [13]. It is therefore inconceivable that sex specification in FGCs arises from gene regulation through DNA methylation. However, it is unclear whether histone modifications regulate sex-specific gene expression in FGCs following erasure of DNA methylation and whether this is essential for fFGC and mFGC differentiation. 
Evidence for the involvement of histone modifications in gene regulation and cell differentiation comes from a variety of cell types [14]. Bivalent domains - which are characterized by the coexistence of activating (trimethylation of lysine 4 on histone $\mathrm{H} 3$ [H3K4me3]) and repressive (histone 3 lysine 27 trimethylation [H3K27me3]) histone marks $[15,16]$ - are thought to play an important role in maintaining pluripotency by silencing developmental genes until their activation during differentiation [17]. In the germline some genes are maintained in a primed state from the initiation of sexual differentiation during fetal development until postmeiotic stages [18]. Furthermore, allele-specific resetting of histone marks in mouse embryos [19] has been investigated by a procedures using low number of cells $[19,20]$; however, these methods still need to be optimized.

Epigenetic modifications are closely related to chromatin conformation and the active or repressive state of the genome [21,22]. A recent study evaluated genome-wide chromatin accessibility in human and mouse FGCs [23], and established a genome-wide map of the nucleosome-depleted regions, in which distal regions were enriched with binding motifs of pluripotency and germ cell master regulators.

To gain further insight into the mechanisms underlying sex specification of FGCs and establishment of epigenetic signatures of gametes, we performed a comprehensive analysis of histone modifications in E13.5 FGCs and P1 neonatal germ cells (NGCs) by native chromatin immunoprecipitation and sequencing (ChIP-seq) [21]. We also evaluated chromatin accessibility in these cells. Comparisons between the transcriptome, DNA methylome and chromatin state suggest that a specific combination of histone modifications functions as the major epigenetic regulator of sex specification in GCs following erasure of DNA methylation.

\section{Materials \& methods}

Animals \& PGC collection

Female C57BL/6 mice that were 10-12 weeks old (Clea Japan, Tokyo, Japan) were crossed with male homozygous Pou5f1- $\triangle P E$-green fluorescent protein (GFP) mice (C57BL/6 background) [24]. The day the vaginal plug was first detected was defined as E0.5. Heterozygous Pou5f1- $\triangle P E-G F P$ embryos were recovered at E13.5 and P1, and the sex was distinguished based on the morphology of the gonad. Sex at E10.5 was judged by PCR detection of sex specific genes, Xist for female and $Z f y$ for male, using a part of each embryo. Female and male genital ridges were separately pooled for PGC and FGC sorting. The gonad was treated with a $1 \mathrm{mg} / \mathrm{ml}$ collagenase solution (Wako Pure Chemical Industries, Osaka, Japan) at $37^{\circ} \mathrm{C}$ for $40 \mathrm{~min}$, followed by treatment with $0.25 \%$ trypsin-EDTA solution ( $0.53 \mathrm{mM}$; Sigma-Aldrich, MO, USA) at $37^{\circ} \mathrm{C}$ for $15 \mathrm{~min}$. After adding fetal bovine serum, a single-cell suspension was obtained by gentle pipetting. GFP-positive PGCs and FGCs were isolated by fluorescence-activated cell sorting (FACS) with a FACSAria II cell sorter (BD Biosciences, NJ, USA).

This study was carried out in strict accordance with the Tokyo University of Agriculture Guide for the Care and Use of Laboratory Animals. The protocol was approved by the Committee on the Ethics of Animal Experiments of the Tokyo University of Agriculture (permit no. 260064SE). Animals were sacrificed by cervical dislocation for sample collection.

\section{ChIP-seq}

PGCs, FGCs and NGCs were collected from the gonads of Pou 5 f1- $\triangle P E-G F P$ mouse embryos and pups and were sorted on a FACSAria II system, and then preserved in Nuclei EZ Lysis Buffer (Sigma-Aldrich; N3408). A total of 10,000 (FGCs and NGCs) or 4000 (E10.5 PGCs) cells were pooled and digested with micrococcal nuclease (New England Biolabs, MA, USA; M0247S) for each ChIP reaction. The following antibodies were used in this study: rabbit anti-H3K4me3 (\#39915) and rabbit anti-H3K4me1 (\#39297) (both from Active Motif, CA, USA); rabbit anti-H3K9me3 (Diagenode, NJ, USA; C15410193); mouse anti-H3K9me2 (MBL, IL, USA; MABI0317); rabbit anti-H3K27ac (Abcam, MA, USA; ab4729); and rabbit anti-H3K27me3 (Millipore, MA, USA; \#07-449). The suitable antibody for each ChIP analysis was selected from three to five products of different firms by validations of ChIP-qPCR and small scale ChIP-seq. ChIP experiments were performed as previously described [21]. Next-generation sequencing (NGS) libraries were prepared based on the LIMprep2 protocol (http: //bit.riken.jp/protocols/) [25] using the KAPA Hyper Prep kit (KAPA Biosystems, MA, USA; KK8502). NGS libraries were validated with a Bioanalyzer HS system (Agilent Technologies, CA, USA) and quantitative (q)PCR was carried out with the KAPA Library Quantification kit (KAPA Biosystems; KK4824), with sequencing performed using NextSeq500 v.2 (150 cycles) or MiSeq Reagent v.3 (Illumina, CA, USA). 


\section{ATAC-seq}

To map chromatin-accessible sites in the genome, we performed ATAC-seq as previously described using 20,000 freshly collected FGCs and NGCs [26]. For PCR amplification of each sample and library replicate, the linear range of cycle numbers was determined by qPCR and 11-13 cycles of amplification were performed. Amplified libraries were purified with SPRI AMPure XP beads (Beckman Coulter, Brea, CA, USA) and subjected to NGS on NextSeq500 v.2.

\section{ChIP- \& ATAC-seq data analysis}

Sequence tags were aligned to the mouse genome (mm10) using bowtie2.2.3 (http://bowtie-bio.sourceforge.net /bowtie2/index.shtml) [27]. Read1 and Read2 tags generated by paired-end sequencing were merged and mapped as a single read after removing the first and last bases of each read with the bowtie2 function ' $-51-31$.' The sequence features are summarized in Supplementary Tables $1-5$. Spearman's rho correlation coefficients in $500-\mathrm{kb}$ bins were calculated with deepTools2 [28] excluding sex chromosomes, ENCODE blacklist regions and overcounted bins using the '-removeOutliers' option. All mapped reads were converted to their 3 ' end (ChIP-seq) or 5' end (ATAC-seq) single base, and merged duplicate library sequences were used for subsequent analyses. The read density of bins across the chromosome were calculated with the bedtools [29] intersect command and normalized according to the total number of mapped reads. To evaluate histone modification/open chromatin profiles around gene loci and CGI regions, the seqMINER platform was used to collect the read numbers of these regions [30].

\section{Peak definition for H3K4me3 ChIP-seq data}

Peak calling was performed with MACS2 (2.1.1.20160309) [31] using the '-nomodel' option. Merged duplicate sequences of full-length reads were used for MACS2 peak calling analysis. Peaks in sex chromosomes and overrepresented regions were excluded; the latter contained ENCODE blacklist regions and common regions of peaks that were called in our three ChIP input libraries by MACS2. To extract regions that were differentially enriched in females and males, overlapping peaks were merged and reads located in each peak region were counted. All reads numbers in the peaks in the peak regions were normalized and compared by DESeq2 [32]. Peaks with a value of 1 or greater for the $\log 2$ fold change and greater that $500 \mathrm{fpk}$ read density were identified as differentially accumulated peaks.

\section{Bivalent domains around transcription start sites in FGCs}

Bivalent domains are the regions bearing both activation and repression histone marks, so we classified the degree of $\mathrm{H} 3 \mathrm{~K} 4 \mathrm{me} 3 / \mathrm{H} 3 \mathrm{~K} 27 \mathrm{me} 3$ enrichment around the transcription start site (TSS) region of 28,025 RefSeq genes by K-means clustering (ranked). Using the number of $\mathrm{H} 3 \mathrm{~K} 4 \mathrm{me} 3$ reads in the window from $-0.5 \mathrm{~kb}$ upstream to $0.5 \mathrm{~kb}$ downstream of RefSeq genes TSSs, loci were grouped into six clusters; regions in one of the three clusters showing a high accumulation of reads were categorized as being enriched in genes bearing $\mathrm{H} 3 \mathrm{~K} 4 \mathrm{me} 3$ modifications. These H3K4me3-marked genes (13,679 in female and 13,560 in male) were reorganized into eight clusters by the number of $\mathrm{H} 3 \mathrm{~K} 27 \mathrm{me} 3$ reads in the $\pm 2 \mathrm{~kb}$ shore region of the TSS. K-means clustering and heatmap visualization were performed with the seqMINER platform [30].

\section{RNA-seq}

Total RNA was purified from 2000 P1 GCs using the RNeasy Micro kit (Qiagen, CA, USA). cDNA was synthesized and amplified with the SMART-Seq v.4 Ultra Low Input RNA kit (Clontech, CA, USA). Double-stranded cDNA was sheared into 200-bp fragments using the Adaptive Focused Acoustics system (Covaris, MA, USA), and sequencing libraries were established using the KAPA Hyper Prep kit. The 50-bp single-read tags were trimmed by removing three bases from the $5^{\prime}$ end and one from the $3^{\prime}$ end, and were analyzed with the TopHat/Cufflinks pipeline in order to quantify transcript levels [33].

\section{CGI clustering}

CGI clusters were generated according to average DNA methylation levels at each CpG site across the CGI. $\mathrm{K}$-means clustering was performed using the $\mathrm{R}$ package 'kmeans' function $(\mathrm{k}=8)$. Heatmaps of ChIP-seq and ATAC-seq read distributions were visualized with the seqMINER platform [30]. 


\section{Data access}

All sequencing data in this study can be accessed at DDBJ (DNA Data Bank of Japan) under the accession number DRA006633.

\section{Results}

\section{Histone modifications in FGCs and NGCs}

To obtain a sufficient number of cells for reliable ChIP-seq analysis, we isolated FGCs at E13.5 and NGCs at P1 from a transgenic octamer-binding transcription factor 4-GFP transgenic mouse line by FACS as previously reported [24]. The purity of the germ cells as assessed by immunostaining for the germ cell specific marker DDX4, was $>98 \%$. To obtain reliable data, we optimized both the specific antibody used and its dilution. We then determined the accuracy of our ChIP-seq results by correlation coefficient testing and found that each dataset was highly reproducible (Figure 1A, B \& Supplementary Figure 1). The correlation coefficients differed markedly between FGCs and P1 NGCs as well as between oocytes (P1o) and spermatogonia (P1s). However, there were few differences in histone modifications between E13.5 fFGCs and mFGCs. The number of activating histone marks over a gene region were highly correlated with gene expression levels (Supplementary Figure 2). Additionally, promoters of genes that were preferentially expressed in either fFGCs or mFGCs showed greater enrichment in H3K4me3 and H3K27ac marks (Supplementary Figure 3) [13]. Here, we analyzed histone modifications of NGCs in addition to FGCs, although cell cycle stage of P1 oocytes and P1 spermatogonia are meiotic and mitotic stage, respectively. It is necessary to understand whether the sex differences of histone modifications in FGCs are specific and different from those of NGCs, and to provide a comparison with the DNA methylome data [11,12].

To investigate these correlations in greater detail, we performed a peak call analysis for $\mathrm{H} 3 \mathrm{~K} 4 \mathrm{me} 3$ using the MACS2 program (Figure 1C). The results identified 7050 and 5483 H3K4me3 peaks that were differentially accumulated ( $\geq$ twofold change) between fFGCs and mFGCs. Of these, a number of peaks ( $>500$ reads per kb [FPK] of the peak region) exhibiting sex differences were identified in fFGCs $(\mathrm{n}=135)$ and $\mathrm{mFGCs}(\mathrm{n}=54)$. Interestingly, in fFGCs and mFGCs, 19 and $29 \%$ of these peaks, respectively were located within the TSS $\pm 0.5 \mathrm{~kb}$ region (Figure 1D). Furthermore, using our previous transcriptome data, we showed that 651 and 428 genes were specifically expressed in female and male FGCs at E13.5, respectively [13] (Supplementary Figure 4). These results suggest that sex differences in histone modification already exist in E13.5 FGCs in which DNA methylation marks have been erased [9] and that the modifications are involved in gene expression in these cells.

Our results raised the question of whether sex differences in histone marks detected in E13.5 FGCs are established immediately before this stage or at earlier time points. To date, there is little information on sex differences in histone modifications in germ line cells at early stages of development. To address this question, we carried out a ChIP-seq analysis of H3K4me3 marks in E10.5 PGCs from gonads at the bisexual stage [4]. We found that the sex-specific H3K4me3 peaks were quite limited in early stage PGCs (Supplementary Figure 5A \& B). Although a small number of E10.5 PGCs were analyzed, the results from this preliminary experiment suggest that sex specific histone modifications are already imposed at some regions even in bisexual stage PGCs. Our findings demonstrated sex-specific histone modifications in E13.5 FGCs, which may be involved in the sex-specific gene expressions. However, illustrating the whole process of sex specification of FGCs by epigenetic mechanisms requires further detailed analysis of this progression with artificial alternations of histone modification states or induced sex reversal.

Next, we generated a genome-wide map (100-kb bin) of histone modifications on chromosome 7 to investigate their overall distribution (Figure 1E). One such important region harboring interesting maternally expressed imprinted genes such as Igf2, H19, are located in the distal region of the chromosome. Interestingly, this imprinted region is regulated by the paternally methylated intergenic DMR. Each of the three activating or repressive histone marks showed similar accumulation across chromosome 7 in fFGCs and mFGCs. However, differences in accumulation patterns were observed between E13.5 FGCs and P1 NGCs in each sex, and also between P1o and P1s. Notably, activating and repressive marks showed opposite accumulation patterns in many regions. Furthermore, $\mathrm{H} 3 \mathrm{~K} 9 \mathrm{me} 3$ was frequently detected in the proximal region, whereas $\mathrm{H} 3 \mathrm{~K} 27 \mathrm{me} 3$ and $\mathrm{H} 3 \mathrm{~K} 9 \mathrm{me} 2$ were distributed throughout the chromosome. The chromatin state as determined by ATAC-seq is dynamic during embryonic development, with open chromatin often containing transcriptional units. The heatmap generated by ATAC-seq enrichment analysis of chromosome 7 showed that the open (high enrichment) and closed (low enrichment) regions formed distinct patterns in each sample (Figure 1E). Sex differences of the patterns were clear in P1 NGCs but limited in FGCs. For example, accessible sites were present at a high density in the distal region in P1o, whereas in P1s the distal region showed a closed conformation. However, in some regions (blue shaded area in male P1 
(A)

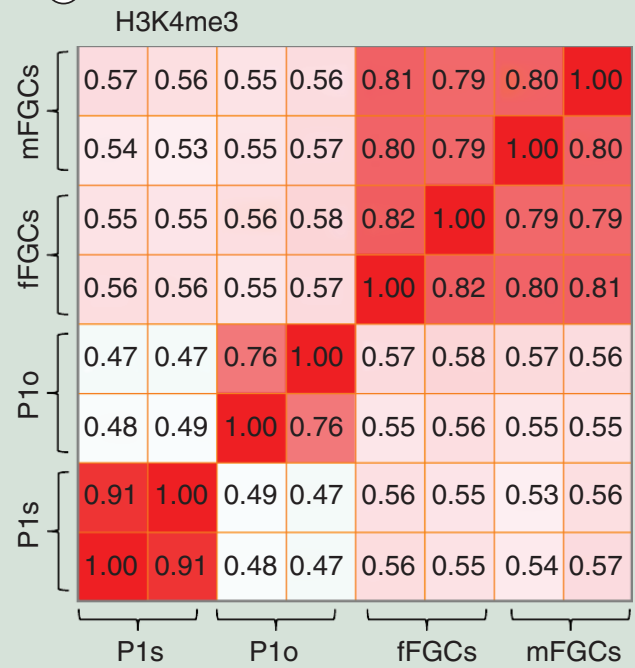

(B)

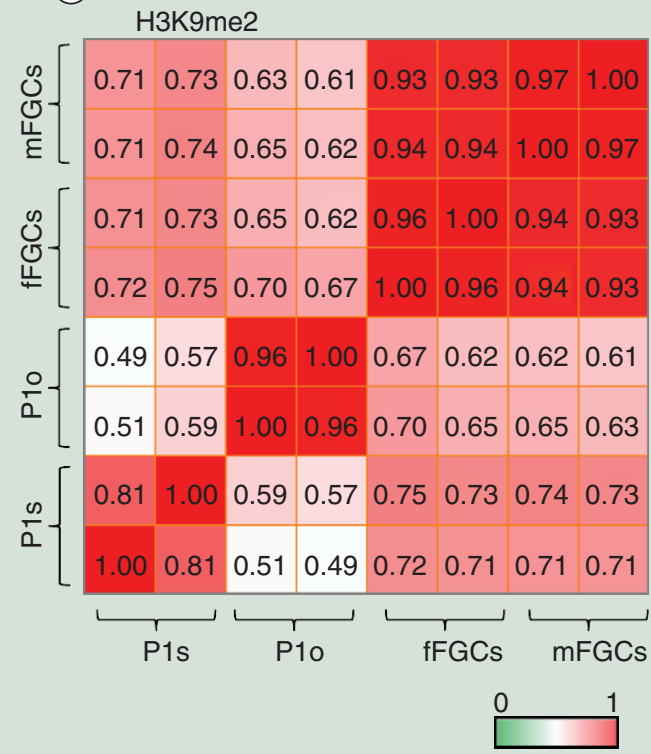

(C)

\begin{tabular}{ccc}
$\begin{array}{c}\text { Total peak } \\
\text { numbers }\end{array}$ & $\begin{array}{c}\text { Differentially } \\
\text { accumulated } \\
\text { peak numbers }\end{array}$ & $\begin{array}{c}\text { Peaks over } \\
500 \mathrm{fpk} \text { in the } \\
\text { peak region }\end{array}$ \\
64,503 & 7050 & 135 \\
\hline 59,828 & 5483 & 54 \\
\hline
\end{tabular}

(D)

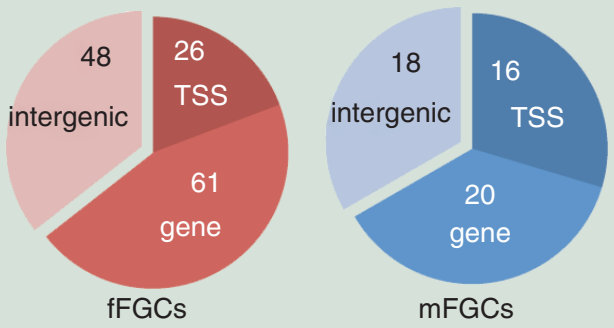

Figure 1. Sex differences in histone modifications. (A \& B) Heatmaps of Spearman's rho correlation coefficients for H3K4me3 (A) and H3K9me2 (B) between fFGCs, mFGCs, P1o and P1s. (C) Number of H3K4me3 peaks differentially enriched in fFGCs and mFGCs, as determined by MACS2. fpk, fragments per kbp. (D) Pie charts displaying the number of differentially accumulated H3K4me3 peaks in each genomic region. Peaks located within the 500-bp region upstream of the transcription start site were classified as transcription start site. (E) Relationship between histone modifications and transposase-accessible chromatin across chromosome 7. Line graphs (100-kb bins) represent histone modifications; the upper and lower panels for each sample show repressive and activating histone modifications, respectively. Bars in the color gradient chart bars (100-kb bins) represent transposase-accessible chromatin sites identified by ATAC-seq. Blue shadows in the male P1s panel show regions with a high accumulation of transposase-accessible sites. These regions were enriched in repressive histones marks, unlike the corresponding regions in fFGCs, mFGCs and P1o, which were enriched in activating histone marks. The top black and white bar represents gene density of chromosome 7. (F) Genomic annotation results from ChIP- and ATAC-seq analyses. Reads in repetitive regions were annotated according to the class of repetitive sequence (http://hgdownload.cse.ucsc.edu/goldenpath/mm10/bigZips/chromOut.tar.gz); the proportion relative to the total mapped reads is shown.

panel, Figure 1E), ATAC-seq enrichment was positively correlated with activating histone marks, which was also revealed by the whole-genome analysis (Supplementary Figure 6A). The highest value (0.62) was associated with H3K4me3 in fFGCs, mFGCs and P1o. The scatterplots showed that activating but not repressive histone marks were correlated with enriched sites identified by ATAC-seq (Supplementary Figure 6B). This finding was confirmed at each enrichment site, and an example is shown in Supplementary Figure 6C. Genomic Regions Enrichment of Annotations Tool analysis revealed many GO terms related to epigenetic gene regulation, among which were sex-specific terms were included (Supplementary Figure 7).

There was comparable accumulation of histone marks at repetitive sequences in the two sexes; however, the ratio of each repeat sequence in the genome varied (Figure $1 \mathrm{~F}$ ). $\mathrm{H} 3 \mathrm{~K} 9 \mathrm{me} 3$ was enriched at satellite sequences in both FGCs and NGCs. High levels of repressive marks - especially H3K9me2 - were detected at long interspersed 


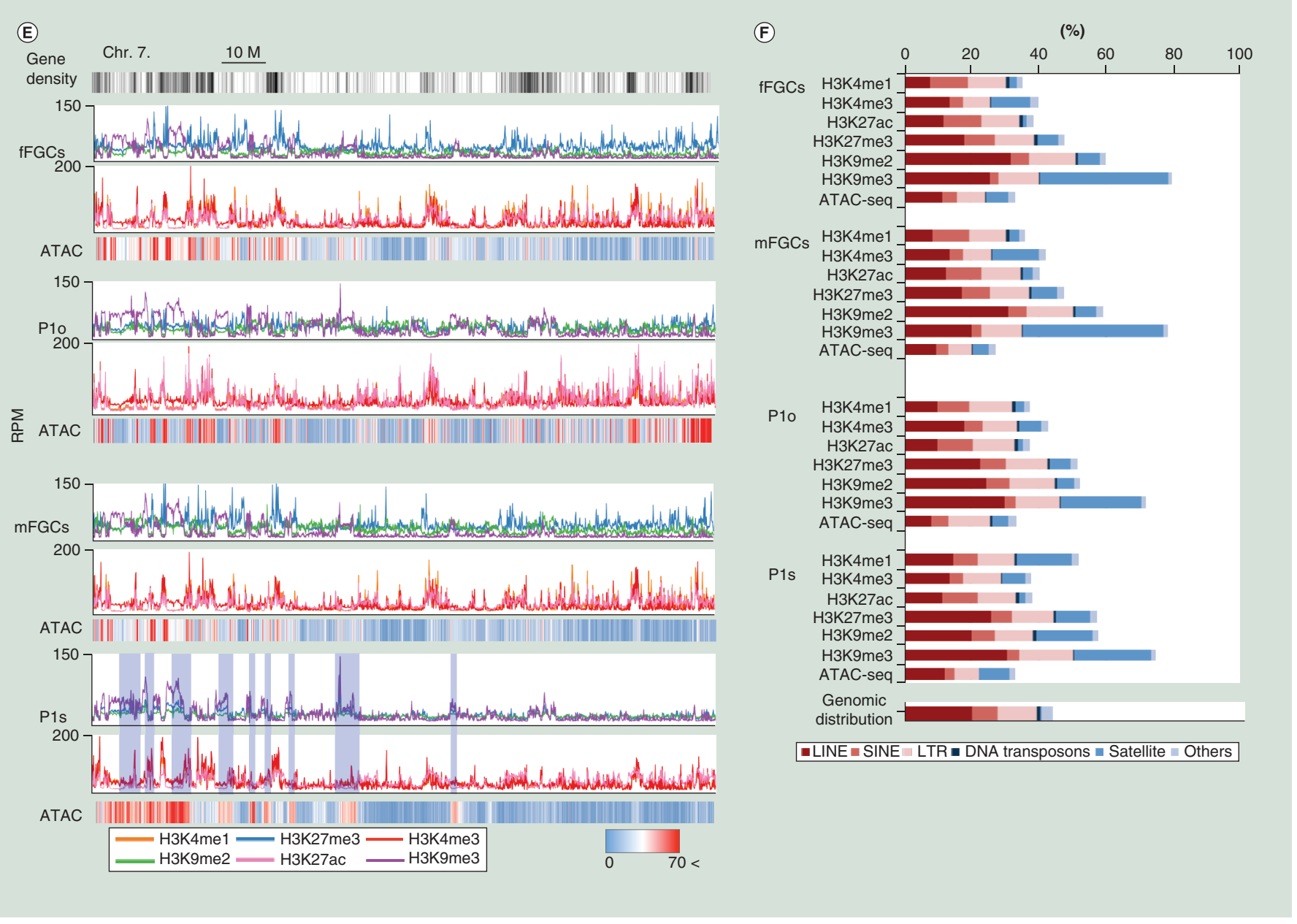

Figure 1. Sex differences in histone modifications (cont.). (A \& B) Heatmaps of Spearman's rho correlation coefficients for H3K4me3 (A) and H3K9me2 (B) between fFGCs, mFGCs, P1o and P1s. (C) Number of H3K4me3 peaks differentially enriched in fFGCs and mFGCs, as determined by MACS2. fpk, fragments per kbp. (D) Pie charts displaying the number of differentially accumulated H3K4me3 peaks in each genomic region. Peaks located within the 500-bp region upstream of the transcription start site were classified as transcription start site. (E) Relationship between histone modifications and transposase-accessible chromatin across chromosome 7. Line graphs (100-kb bins) represent histone modifications; the upper and lower panels for each sample show repressive and activating histone modifications, respectively. Bars in the color gradient chart bars (100-kb bins) represent transposase-accessible chromatin sites identified by ATAC-seq. Blue shadows in the male P1s panel show regions with a high accumulation of transposase-accessible sites. These regions were enriched in repressive histones marks, unlike the corresponding regions in $\mathrm{fFGCs}, \mathrm{mFGCs}$ and $\mathrm{P} 1 \mathrm{o}$, which were enriched in activating histone marks. The top black and white bar represents gene density of chromosome 7. (F) Genomic annotation results from ChIP- and ATAC-seq analyses. Reads in repetitive regions were annotated according to the class of repetitive sequence

(http://hgdownload.cse.ucsc.edu/goldenpath/mm10/bigZips/chromOut.tar.gz); the proportion relative to the total mapped reads is shown.

nuclear elements (LINEs). H3K4me1, an activating enhancer mark, was enriched at satellite repeats in P1s; the reason for this is currently unclear.

\section{Bivalent histone modifications}

Co-localization of $\mathrm{H} 3 \mathrm{~K} 4 \mathrm{me} 3$ and $\mathrm{H} 3 \mathrm{~K} 27 \mathrm{me} 3$ at the promoter region is thought to keep the gene expression repressed until activation is needed for the cell differentiation [17]. To evaluate $\mathrm{H} 3 \mathrm{~K} 4 \mathrm{me} 3 / \mathrm{H} 3 \mathrm{~K} 27 \mathrm{me} 3$ bivalency at promoter regions, we first identified 13,679 and 13,560 genes in fFGCs and mFGCs, respectively, with H3K4me3 accumulation within $\pm 0.5 \mathrm{~kb}$ of the TSS; of these, 2768 and 2970 genes, respectively, showed H3K27me3 enrichment within $\pm 2 \mathrm{~kb}$ of the TSS and were therefore identified as bivalent genes (Figure 2A). Compared with the transcriptome heatmap, it was clear that these bivalent I (high enrichment of H3K4me3/H3K27me3) and II (high and middle enrichment of H3K4me3/H3K27me3) genes were repressed in FGCs as evidenced by their 


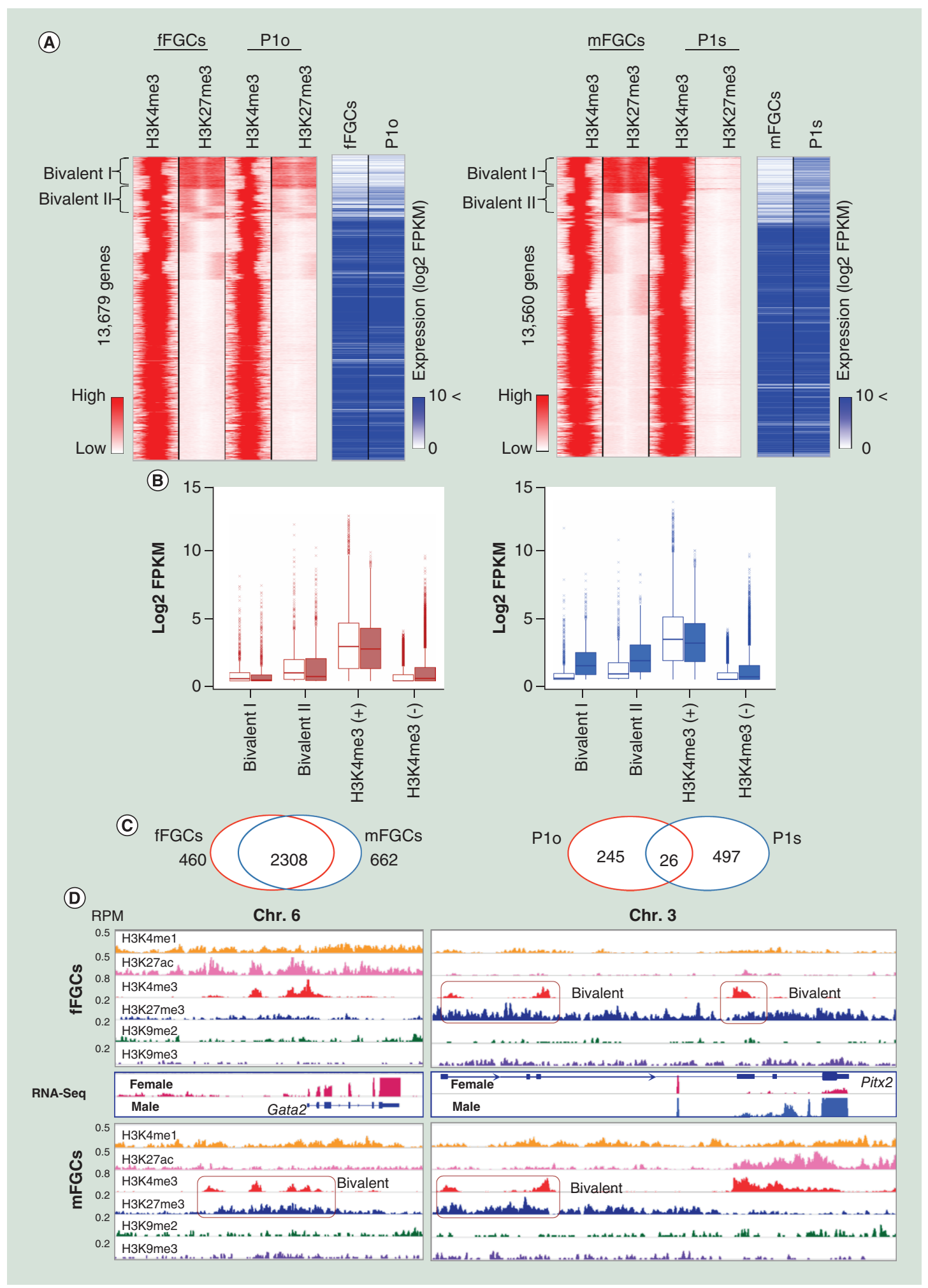

Figure 2. Expression of genes with bivalent histone modification (H3K4me3 and H3K27me3). Bivalent domains around promoters in fetal germ cells (FGCs) are shown. (A) Heatmap of H3K4me3 and H3K27me3 profiles $2 \mathrm{~kb}$ upand downstream of the TSS (red) and gene expression levels (blue) of H3K4me3-positive genes in FGCs. Clusters were generated based on FGC ChIP-seq datasets. (B) Boxplots showing RNA expression levels of genes in each cluster in FGCs (uncolored) and P1 neonatal germ cells (NGCs) (shadowed) in both females (left) and males (right). (C) Venn diagrams showing the number of bivalent genes specific to fFGCs and mFGCs (left) and P1 NGCs (right). Numbers of sex-specific bivalent genes in FGCs and P1 NGCs are shown. D) (D) Screen shot of the Integrative Genomics Viewer browser [52] showing histone modifications and RNA-seq results of regions close to representative genes specifically expressed in fFGCs [Gata2 [A]) and mFGCs (Pitx2 [B]). The area surrounded by a purple frame shows bivalent modifications (H3K4me3 and H3K27me3). 
downregulation. In mFGCs, H3K27me3 was strongly enriched in bivalent I genes; in contrast, these genes in P1s had almost lost the H3K27me3 marks and showed enhanced expression. Therefore, H3K27me3 in bivalent genes has a repressive function. The results of $\mathrm{k}$-means clustering were supported by a boxplot analysis of bivalent gene expression (Figure 2B). The expression levels of bivalent I genes in both fFGCs and mFGCs were similar to those of genes lacking $\mathrm{H} 3 \mathrm{~K} 4 \mathrm{me} 3$.

Interestingly, $460(16.6 \%)$ and $662(22.3 \%)$ bivalent genes were specific to fFGCs and mFGCs, respectively, however, a considerable number of genes $(n=2,308)$ showed bivalency in both (Figure 2C). A Gene Ontology analysis revealed the terms 'meiotic cell cycle process', and 'DNA methylation involved in gamete generation' in fFGCs, and 'cell adhesion', 'cell proliferation' and 'response to chemical' in mFGCs (Supplementary Figure 8). The number of sex specific bivalent genes was diminished in P1o $(n=245)$ and P1s $(n=497)$, with only 26 shown as common to both (Figure 2C). The reduction was mainly caused in the bivalent genes observed in both sex due to decreases in both histone modifications in P1o and in the H3K27me3 mark in P1s. These novel findings suggest that gene silencing by bivalency is reduced as germ cell differentiation progresses.

We next investigated whether the bivalency observed in FGCs was maintained in P1 NGCs. By P1, many of the genes had lost this feature: only $183(6.6 \%)$ genes in fFGCs and $149(5.0 \%)$ in mFGCs were bivalent. However, the number of new bivalent genes was over four times greater in P1s as compared with P1o (374 vs. 88). The regulation of bivalency was associated with fFGC-specific expression of GATA-binding protein 2 (Gata2), a zinc-finger transcription factor involved in the development and proliferation of hematopoietic and endocrine cells (Figure 2D, left). Bivalency was detected solely in mFGCs in which Gata2 was repressed. On the contrary, the mFGC-specific gene paired-like homeodomain transcription factor 2 (Pitx2) was bivalent at exons 4-6 only in fFGCs. Interestingly, the gene was expressed from exon 4 in mFGCs, while exons 1-3 had bivalent modifications in both sexes (Figure 2D, right) and no expression was detected in either fFGCs or mFGCs. We further investigated the relationship between bivalency and sex-specific gene expression. Of the 651 and $428 \mathrm{fFGC}$ - and mFGC-specific genes identified in our previous study [13], eight and 42, respectively, were bivalent. Interestingly, the promoters of sex-specific genes expressed in either fFGCs or mFGCs showed greater enrichment in H3K4me3 and H3K27ac marks (Supplementary Figure 3); meanwhile, there was limited accumulation of H3K4me3 marks in the gene body. Although repressive marks were enriched in gene body regions in both fFGCs and mFGCs, there were no clear differences between the sexes.

\section{Characteristics of repressive histone modifications}

$\mathrm{H} 3 \mathrm{~K} 27 \mathrm{me} 3, \mathrm{H} 3 \mathrm{~K} 9 \mathrm{me} 2$ and $\mathrm{H} 3 \mathrm{~K} 9 \mathrm{me} 3$ act as repressive marks, and $\mathrm{H} 3 \mathrm{~K} 9 \mathrm{me} 2$ and $\mathrm{H} 3 \mathrm{~K} 9 \mathrm{me} 3$ are broadly distributed throughout the genome without a sharp peak. To understand the interrelationship between these three repressive histone marks is important, therefore we investigated their distribution in greater detail and carried out a hidden Markov model (HMM) analysis using chromHMM (Figure 3) [34]. The patterns of repressive histone modifications were segmented into 8 classes (Figure 3A \& B). First, we calculated the CpG methylation levels at each segment using our previous DNA methylome data (DDBJ accession number: DRA000607) [10,12]. Interestingly, the methylation level in each cluster was significantly higher $(\mathrm{p}<0.001)$ in mFGCs than in fFGCs (Figure 3A). Additionally, the methylation level in each cluster was varied between classes and gradually increased from class 1 to class 7 : fFGCs, $1.8-14 \%$ and mFGCs, $2.5-17 \%$.

The majority of genes and the associated regions in both fFGCs and mFGCs were highly modified with $\mathrm{H} 3 \mathrm{~K} 27 \mathrm{me} 3$ (class1) while, in contrast, $\mathrm{H} 3 \mathrm{~K} 9 \mathrm{me} 2$ (class 4), H3K9me3 (class 7) or both (class 6) were sparse (Figure 3C \& D). Most genomic regions were lacking the three repressive marks in both fFGCs and mFGCs. Interestingly, regions marked with $\mathrm{H} 3 \mathrm{~K} 27 \mathrm{me} 3$ and $\mathrm{H} 3 \mathrm{~K} 9 \mathrm{me} 2$ (class 3), especially RefSeq Exon and RefSeq TSS regions, were more enriched in fFGCs compared with mFGCs (Figure 3C \& D). CpG islands showed slightly higher levels of $\mathrm{H} 3 \mathrm{~K} 27 \mathrm{me} 3+\mathrm{H} 3 \mathrm{~K} 9 \mathrm{me} 3$ (class 8) marks in mFGCs than that in fFGCs.

Next, we focused on TSS and TES (transcription end site) regions. Regions from the TSS to 800 bp (Figure 3E $\& \mathrm{~F}$ ) and the TES to -1800 bp (Figure 3G \& H) showed high levels of H3K27me3 and H3K9me2 in fFGCs. Regions marked with $\mathrm{H} 3 \mathrm{~K} 9 \mathrm{me} 2$ (class 4), $\mathrm{H} 3 \mathrm{~K} 9 \mathrm{me} 3$ (class 7) or both (class 6) were lacking the enrichment in the TSS $\pm 2 \mathrm{~kb}$ and TES $\pm 2 \mathrm{~kb}$ regions in all cell types (Figure $3 \mathrm{E}-\mathrm{H}$ ). However, these repressive histone marks were present throughout the gene body excepting TSS and TES regions, and a clear negative correlation between the gene expression level and the accumulation of histone marks was found (Figure 3I). These distributions of repressive histone marks may play a role in promoting stable gene expression. 


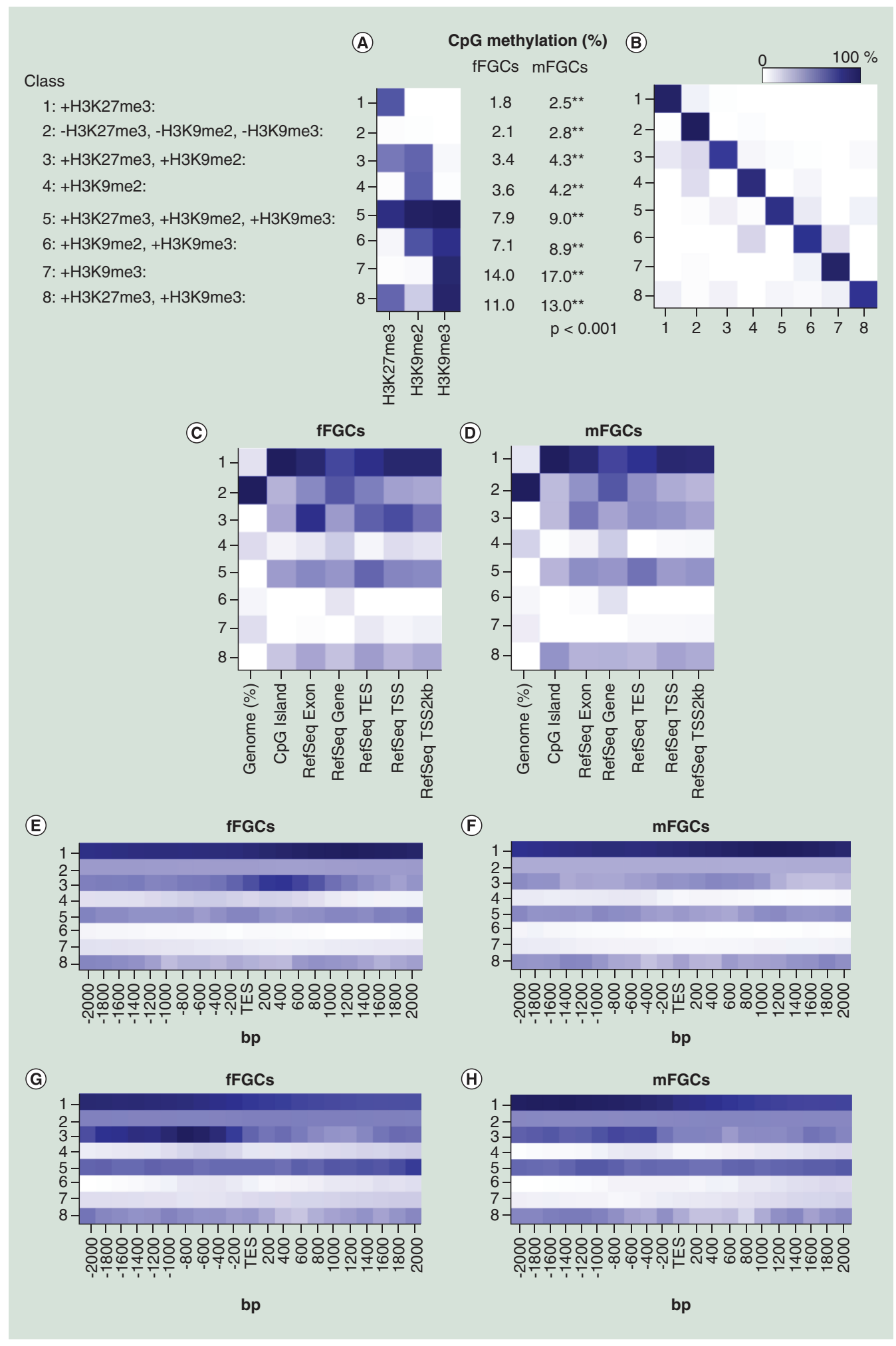

Figure 3. Genomic properties of H3K27me3, H3K9me2 and H3K9me3 modifications by chromHMM analysis. (A) Emission parameters. Asterisks indicate statistically significant difference of DNA methylation at each class between fFGCs and mFGCs (Student's t-test, $p<0.001$ ). (B) Transition parameters. (C \& D) Enrichment of H3K27me3, H3K9me2 and H3K9me3 modifications in specific gene regions in fFGCs (C) and mFGCs (D). (E-H) Enrichment of H3K27me3, H3K9me2 and H3K9me3 modifications adjacent to the TSS (E \& F) and TES (G \& H) region in fFGCS (E \& G) and $\mathrm{mFGCs}(\mathrm{F} \& \mathrm{H})$. (I) Relationship between repressive histone modifications in the gene body region and gene expression levels in fetal germ cells. All protein-coding genes $(n=20,520)$ were divided into four classes: those generating no transcript according to the RNA-seq data [13] were classified as 'not detected', and the others were equally divided into three classes in order to their expression levels (high, moderate and low). 


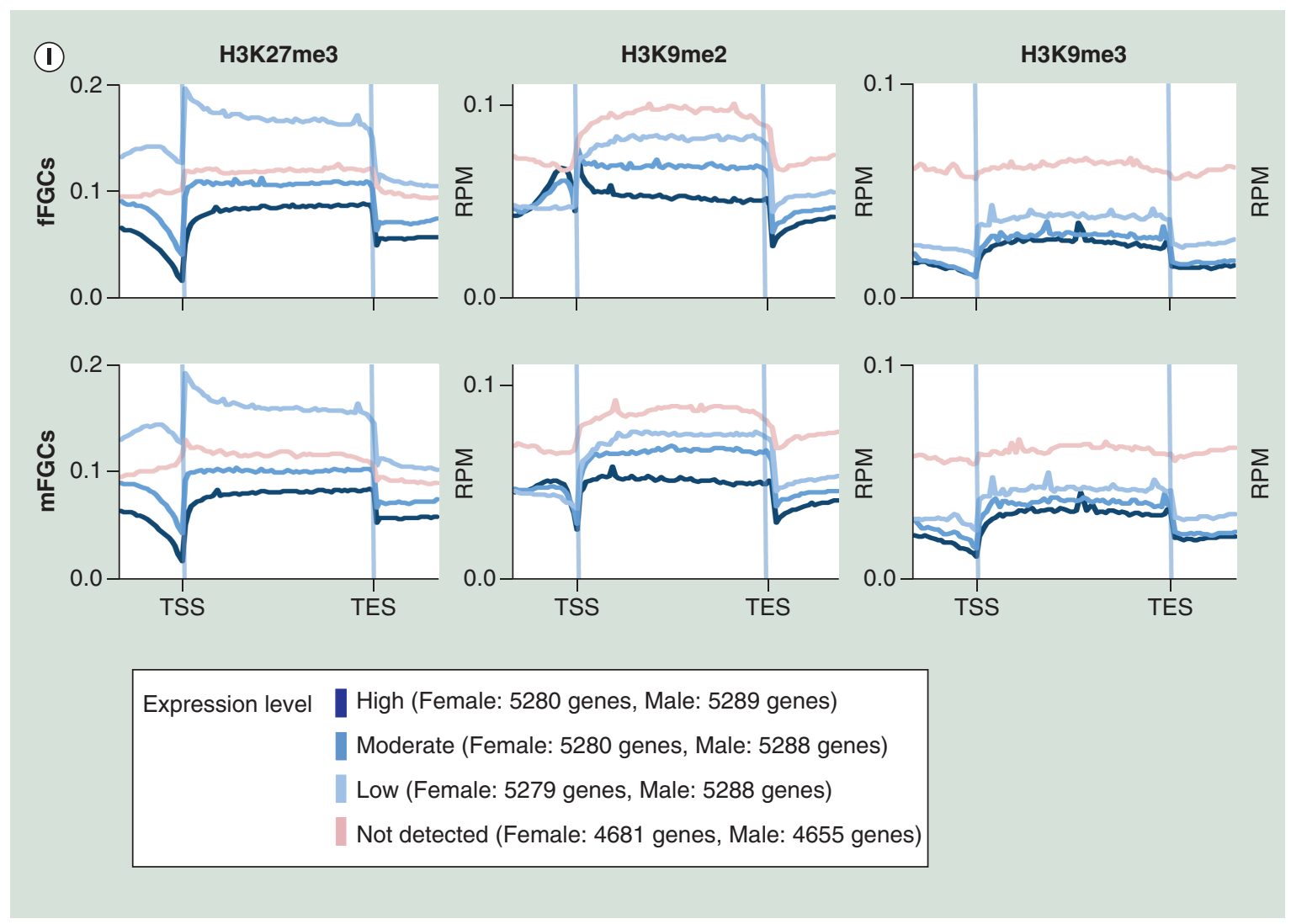

Figure 3. Genomic properties of H3K27me3, H3K9me2 and H3K9me3 modifications by chromHMM analysis (cont.). (A) Emission parameters. Asterisks indicate statistically significant difference of DNA methylation at each class between fFGCs and mFGCs (Student's t-test, $p<0.001$ ). (B) Transition parameters. (C \& D) Enrichment of H3K27me3, H3K9me2 and H3K9me3 modifications in specific gene regions in fFGCs (C) and mFGCs (D). (E-H) Enrichment of H3K27me3, H3K9me2 and H3K9me3 modifications adjacent to the TSS (E \& F) and TES (G \& H) region in fFGCS (E \& G) and $\mathrm{mFGCs}(\mathrm{F} \& \mathrm{H})$. (I) Relationship between repressive histone modifications in the gene body region and gene expression levels in fetal germ cells. All protein-coding genes $(n=20,520)$ were divided into four classes: those generating no transcript according to the RNA-seq data [13] were classified as 'not detected', and the others were equally divided into three classes in order to their expression levels (high, moderate and low).

\section{Histone modifications in CGIs}

A total of 23,021 CGIs have been identified in the mouse genome, some of which are function as regulatory domains for gene expression [35]. We examined the enrichment of histone marks at CGIs to clarify the relationship between histone modification and CGI methylation status. Each histone modification observed in heatmaps of the CGI $\pm 5 \mathrm{~kb}$ region was associated with a characteristic CGI methylation pattern (Figure 4A). DNA methylome data (DDBJ accession numbers: DRA000607, DRA000484, DRA002402) were obtained from our previous study $[10,12]$. H3K4me3 was specifically enriched in CGIs, except in P1s and H3K27ac showed the same tendency as $\mathrm{H} 3 \mathrm{~K} 4 \mathrm{me} 3$. H3K4me1 accumulation was detected in regions adjacent to CGIs, except P1o type II and III regions where the mark was enriched to CGIs regions. Meanwhile, repressive histone marks tended to accumulate in regions surrounding CGIs, except for $\mathrm{H} 3 \mathrm{~K} 9 \mathrm{me} 2$ in fFGCs. $\mathrm{H} 3 \mathrm{~K} 9 \mathrm{me} 2$ was randomly distributed in fFGCs, whereas $\mathrm{H} 3 \mathrm{~K} 9 \mathrm{me} 3$ was infrequently detected in $\mathrm{P} 1 \mathrm{o}$.

CGIs were classified as one of the following four types based on the methylation status in germinal vesicle (GV) oocytes and sperm [10]: I, CGIs methylated in both GV oocytes and sperm $(\mathrm{n}=656)$; II, CGIs methylated in GV oocytes only $(n=4,037)$; III, CGIs methylated in sperm only $(n=1,518)$; and IV, non-methylated CGIs $(\mathrm{n}=16,790)$ (Figure 4A). Each CGI methylation cluster demonstrated a specific histone accumulation pattern. Clusters of highly methylated CGIs of types I and IIIa lacked the H3K4me3 mark in all four cell types, and no H3K4me1 accumulation was observed in P1s (Figure 4B). Types II and IV showed similar modification patterns: $\mathrm{H} 3 \mathrm{~K} 4 \mathrm{me} 3$ and $\mathrm{H} 3 \mathrm{~K} 27 \mathrm{ac}$ were enriched at CGIs, while $\mathrm{H} 3 \mathrm{~K} 4 \mathrm{me} 1$ accumulation was observed at either 


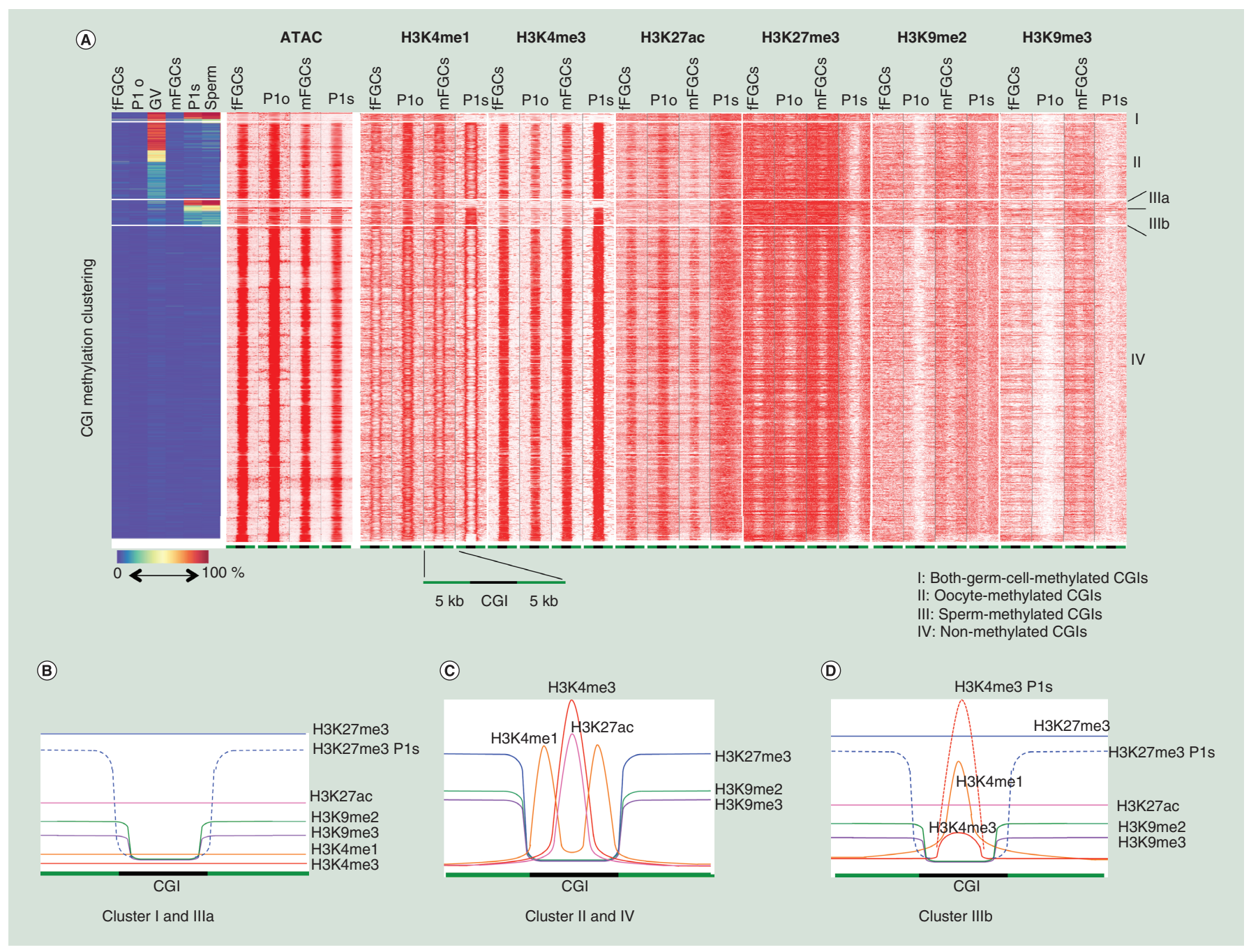

Figure 4. Histone modifications around CGIs. (A) Heatmap of ChIP- and ATAC-seq profiles $5 \mathrm{~kb}$ up- and downstream of the 23,001 CGIs identified by Deaton et al. (2011) [35]. CGls were grouped into the following four clusters based on methylation levels in CGls of developing germ cells (left panel): I, CGIs methylated in both GCs ( $=656)$; II, CGIs methylated in oocytes ( $n=4037)$; III, CGIs methylated in sperm ( $n=1518)$; and IV, non-methylated CGIs $(n=16,790)$. Diagram of histone modification patterns at CGIs and surrounding regions in cluster I and the sub-structure of cluster IIIa (B), clusters II and IV (C) and the sub-structure of cluster IIIb characterized by the co-existence of intermediate DNA methylation and H3K4me3 enrichment (D).

end of CGIs flanking the H3K4me3 and H3K27ac peaks (Figure 4C). Repressive marks were abundant in the regions surrounding CGIs. Striking histone modification patterns were observed in clusters of type III CGIs. A sub-structure IIIb was observed that was characterized by the co-existence of intermediate DNA methylation and H3K4me3 enrichment at the CGI (Figure 4D). Furthermore, H3K4me1 accumulation was shifted to CGIs, while H3K27me3 was distributed throughout the CGI and surrounding regions, except in P1s. We next investigated chromatin accessibility at CGIs based on ATAC-seq data, since open chromatin frequently contains transcriptional units $[23,36]$. We found that chromatin-accessible sites were highly enriched at CGIs (Figure 4A). In addition to H3K4me3 accumulation, hypermethylated cluster of type I and IIIa CGIs in oocytes and sperm lacked accessible sites. These results suggest that H3K4me3 marks at CGIs promote a chromatin-accessible state. Furthermore, we demonstrated that histone accumulation patterns at CGIs and their shore regions ( $\pm 5 \mathrm{~kb}$ ) were distinguished by whether the CGIs were methylated in oocytes, sperm, or both (Figure 4). Our relatively broad analysis did not reveal clear difference between fFGCs and mFGCs; therefore, further detailed comparative analyses are needed to identify latent sex-specific differences. 


\section{Histone modifications in imprinted genes}

The DNA methylation imprint, which ensures the sex-specific features of female and male gametes, is established at imprint control regions (ICRs). Therefore, we investigated histone modification patterns at ICRs of fFGCs and mFGCs and found that they were highly and similarly enriched in $\mathrm{H} 3 \mathrm{~K} 4 \mathrm{me} 3$, suggesting that the chromatin was in an open state (Figure 5A). Notably, some maternally imprinted genes - e.g. paternally expressed gene (Peg) 3 , small nuclear ribonucleoprotein polypeptide $\mathrm{N}(S n r p n)$ and impact RWD domain protein (Impact) - were also marked by H3K27ac. Modification with H3K27ac and H3K4me3 in ICRs was similar in fFGCs and mFGCs.

In contrast to activating histone marks, sex-specific accumulation of $\mathrm{H} 3 \mathrm{~K} 27 \mathrm{me} 3$ and $\mathrm{H} 3 \mathrm{~K} 9 \mathrm{me} 2$ was detected in some ICRs - H3K9me2 was more abundant in ICRs of Grp1, histocompatibility 13 (H13), Peg3, Grb10 and Impact in fPGCs as compared with mPGCs. In addition, in mPGCs, H3K27me3 was more prevalent in ICRs of guanine nucleotide binding protein, alpha stimulating (Gnas), Gnas-neuroendocrine secretory protein antisense (Gnas-Nespas) and solute carrier family 38 member 4 (Slc38a4). These novel findings provide better understanding for epigenetic regulation of imprinted genes in germ line development. Unfortunately, our ChIP-seq analysis is unable to provide data on maternal or paternal allele specific accumulation histone marks due to an inbred strain of mice being used.

Interestingly, histone marks at ICRs were drastically altered at P1 (Figure 5A). H3K4me1 accumulation was enhanced at all ICRs in P1o relative to that observed in FFGCs. In contrast, the accumulation was reduced in P1s relative to that in mFGCs. This trend was also observed for H3K27ac at many ICRs. However, H3K4me3 was highly enriched at the ICRs of Gnas, Gnas-Nespas, Peg10, mesoderm-specific transcript (Mest) and Slc38a4 in mFGCs. Furthermore, in the intergenic differentially methylated region (IG-DMR), the ICRs of Ras-GRF1 and Dlk1-Gtl2 - which are paternally methylated - were modified by $\mathrm{H} 3 \mathrm{~K} 9 \mathrm{me} 2$ and $\mathrm{H} 3 \mathrm{~K} 9 \mathrm{me} 3 \mathrm{in}$ mFGCs. In contrast, the ICR of $\mathrm{H} 19$ showed few modifications. Thus, although fFGCs and mFGCs at E13.5 were not distinguishable based on histone marks at ICRs, sex-specific modifications were established by P1.

As an example of paternally methylated ICRs obtained by our previous reports (DDBJ accession numbers: DRA000484, DRA002402) [10,12], IG-DMR and Gtl2-DMR were segregated to cluster III (CpG island [CGIs] methylated in sperm) and cluster II (CGIs methylated in oocytes), respectively (Figure 5B). Activating histone marks (H3K4me1, H3K27ac and H3K4me3) were similarly enriched in the IG-DMR of fFGCs and mFGCs and were depleted in P1 NGCs of both sexes. In contrast, repressive marks (H3K9me2 and H3K9me3) were abundant in the IG-DMR region of P1s. Notably, the Gtl2-DMR region - which is considered as a secondary DMR - had an abundance of activating marks (H3K4me1 and H3K4me3) in P1s.

\section{Discussion}

Overexpressing the transcription factors, Blimp1, Prdm14 and AP-2 gamma $2 c$ in epiblast-like cells induces their differentiation into PGCs [37]. Deleted in azoospermia-like (DAZL), an RNA-binding protein, is involved in sex specification of PGCs; its absence blocks the differentiation of PGCs into ovarian and testicular GCs and causes them to remain in a sexually undifferentiated state [38]. Using bulk and single-cell transcriptomic approaches we identified genes that are specifically expressed in fFGCs and mFGCs at E13.5 [13]. However, little is known of the epigenetic mechanisms underlying sex specification of PGCs. Considering default of DNA methylation [9], histone marks could be supposed to involve in gene expression of the FGCs. The present results demonstrated that active histone marks, H3K4me3, H3K4me1, H3K27ac, which are often present at TSSs, were detected at high levels in sex-specific genes, whereas recessive marks, H3K9me2, H3K9me3, H3K27me3, showed lower abundance. Furthermore, it was found that sex difference of the histone modifications at TSS and gene regions are present in FGCs. Interestingly these differences are correlate with the gene expression levels. Therefore, we presume that given the default state of DNA methylation, histone modifications are one of epigenetic marks for involving in sex-specific gene expression in FGCs.

To confirm further this we shed light on the bivalency of H3K4me3 and H3K27me3. Bivalent $\mathrm{H} 3 \mathrm{~K} 4 \mathrm{me} 3 / \mathrm{H} 3 \mathrm{~K} 27 \mathrm{me} 3$ histone marks confer flexibility in terms of gene activation or repression for fate determination in undifferentiated cells [17]. It has been previously reported that developmentally regulated genes in FGCs exist in a bivalent state, with activating and repressive marks, H3K4me3 and H3K27me3, and are transcriptionally silent at E13.5 [17]. This bivalency was also mainly detected at partially methylated long terminal repeats and LINE1 elements [39]. Such particular bivalent marks may have complementary functions in gene regulation that are reinforced by other histone modifications [40]. Our analysis showed that $\mathrm{H} 3 \mathrm{~K} 4 \mathrm{me} 3 / \mathrm{H} 3 \mathrm{~K} 27 \mathrm{me} 3$ bivalency occurred in numerous gene regions; furthermore, these coincided with gene repression. Recent work by Hanna 
(A)

FPKM

FGCs

NGCs

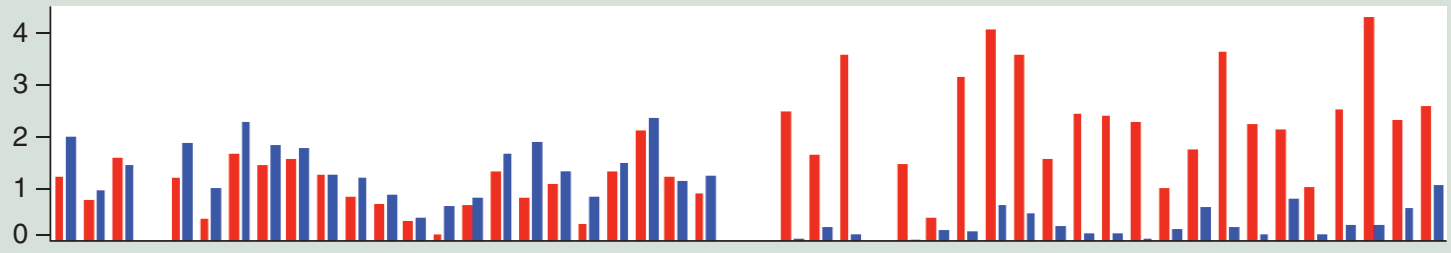

H3K4me1

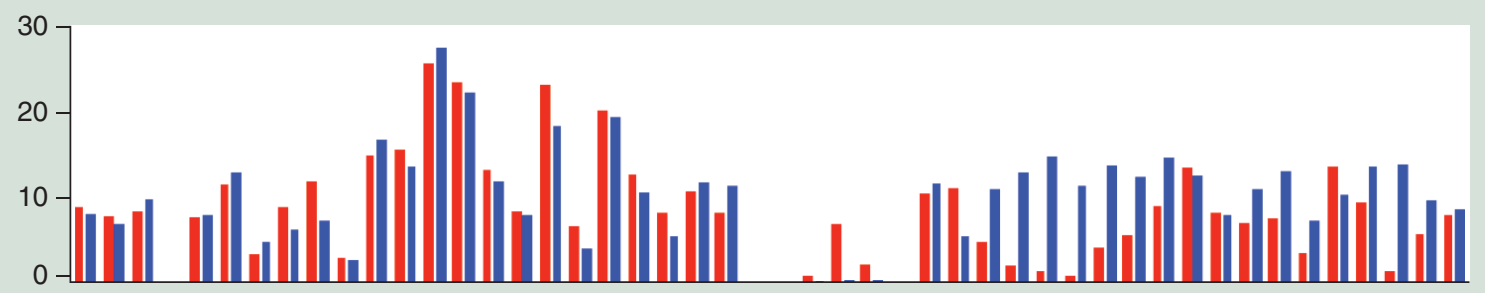

H3K4me3

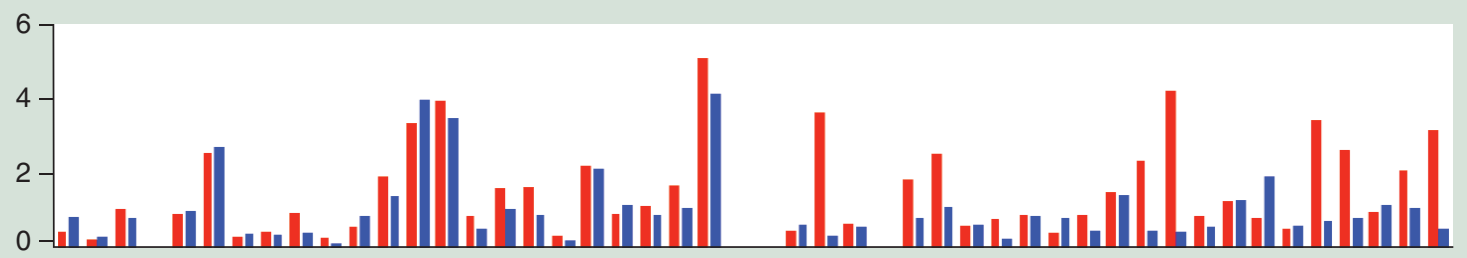

H3K27ac

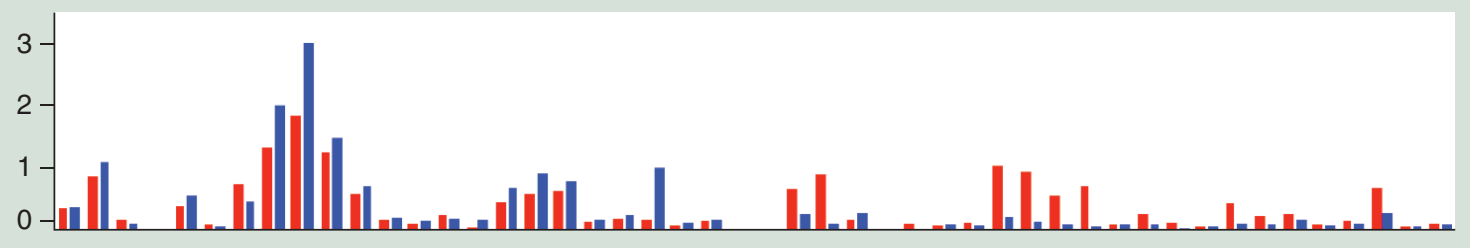

H3K27me3

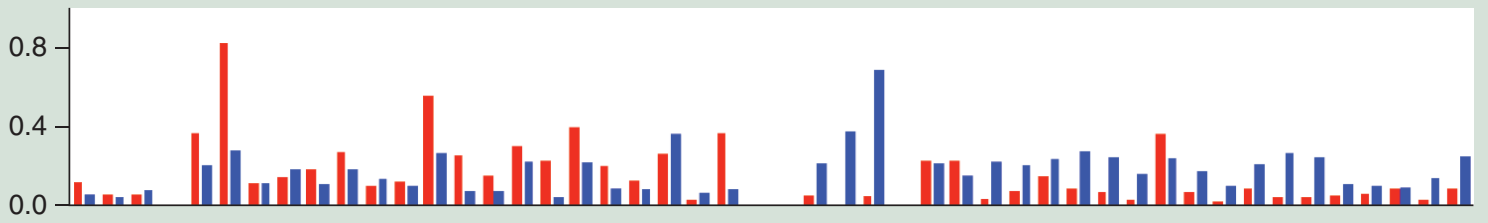

H3K9me2
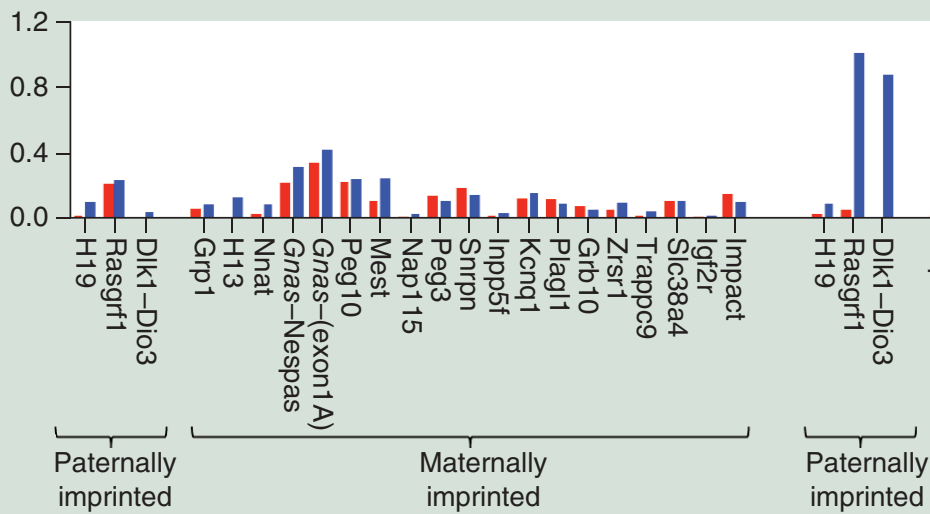

H3K9me3

Figure 5. Histone modifications at imprinting control regions in primodial germ cells and P1 germ cells. (A) Histone marks at imprint control regions in $\mathrm{fFGCs}$ and $\mathrm{mFGCs}$. Enrichment of histone marks at each imprint control region is shown as fragments per kb of imprint control region per million mapped fragments $(\mathrm{fpkm})$. (B) Screen shot of the genome showing histone modifications and DNA methylation level in the distal region of chromosome 12. IG- and Gt/2-DMRs were segregated into clusters II (CpG islands methylated in oocytes) and III (CpG islands methylated in sperm), respectively, in Figure 4A.

DMR: Differentially methylated region; FGC: Mouse fetal germ cell; NGC: Neonatal germ cell. 


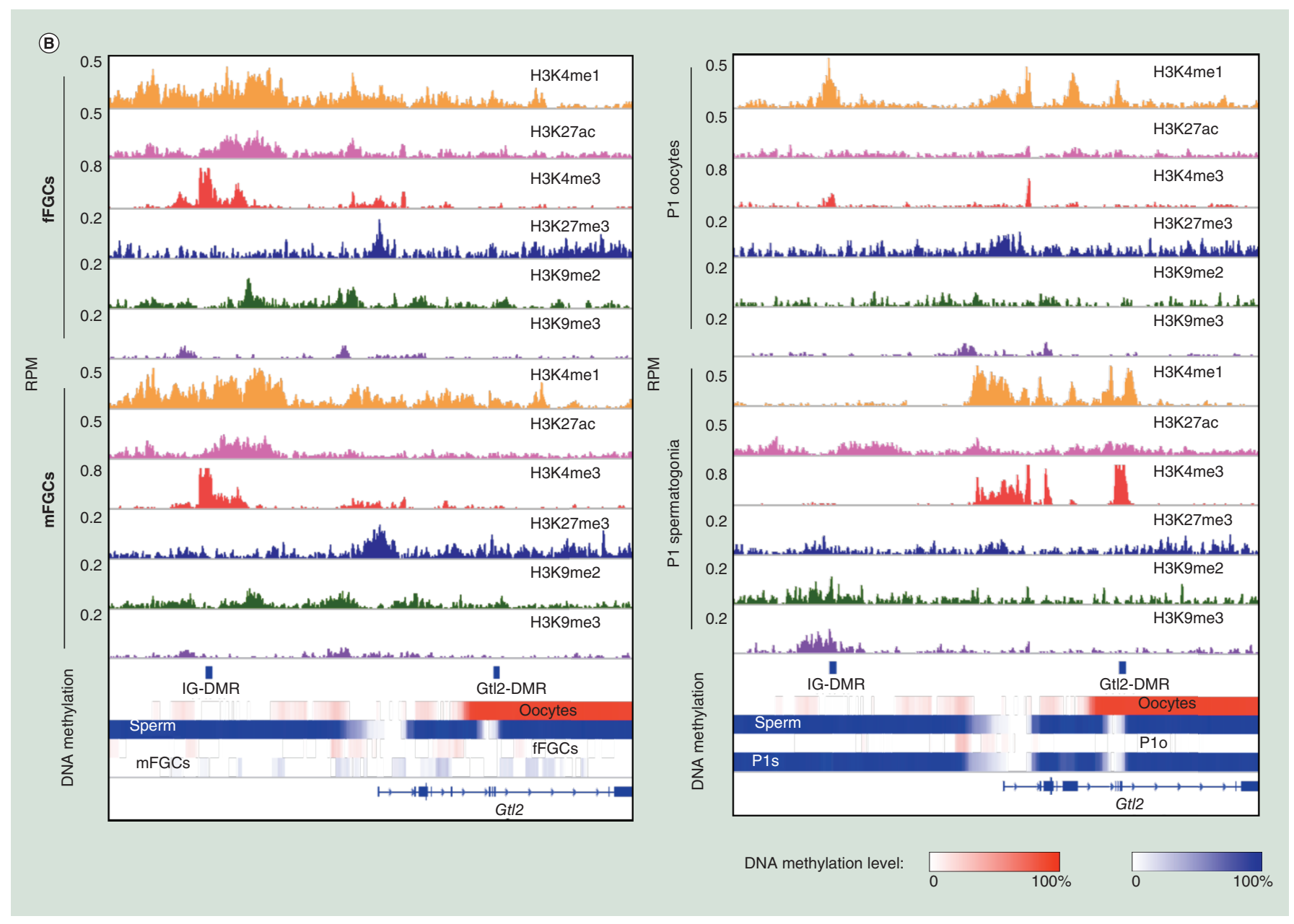

Figure 5. Histone modifications at imprinting control regions in primodial germ cells and P1 germ cells (cont.). (A) Histone marks at imprint control regions in $\mathrm{fFGCs}$ and $\mathrm{mFGCs}$. Enrichment of histone marks at each imprint control region is shown as fragments per kb of imprint control region per million mapped fragments (fpkm). (B) Screen shot of the genome showing histone modifications and DNA methylation level in the distal region of chromosome 12. IG- and Gt/2-DMRs were segregated into clusters II (CpG islands methylated in oocytes) and III (CpG islands methylated in sperm), respectively, in Figure 4A.

DMR: Differentially methylated region; FGC: Mouse fetal germ cell; NGC: Neonatal germ cell.

et al. (2018) [41] reported that the total number of bivalent domains in day 5 nongrowing oocytes was less than 300 [41]. Although precise comparison with their data sets has not been done, taken together with our findings, these data contradict the hypothesis that bivalent domains throughout the germline are maintained in the embryo, as was asserted by Lesch $e$ al. [18]. Further, we investigated the relationship between bivalency and sex-specific gene expression. Of the 651 and $428 \mathrm{fFGC}$ - and mFGC-specific genes identified in our previous study [13], eight and 42, respectively, were bivalent. Interestingly, the promoters of sex-specific genes expressed in either fFGCs or mFGCs showed greater enrichment in H3K4me3 and H3K27ac marks (Supplementary Figure 3); meanwhile, there was limited accumulation of $\mathrm{H} 3 \mathrm{~K} 4 \mathrm{me} 3$ marks in the gene body. Although repressive marks were enriched in gene body regions in both fFGCs and mFGCs, there were no clear differences between the sexes.

An important issue that has not been clarified in this research is the overall interrelationship of epigenetic modifications underlying regulation of gene expression. However, further precise investigations and development of highly accurate analysis methods will be conducted to further understanding of this.

Our results raised the question of whether sex differences in histone marks detected in E13.5 FGCs are established immediately before this stage or at earlier time points. To date, there is little information on sex differences in histone modifications in germ line cells at early stages of development. To address this question, we carried out a ChIP-seq analysis of H3K4me3 marks in E10.5 PGCs from gonads at the bisexual stage [4]. Contrary to our 
expectation, we observed a clear difference in H3K4me3 accumulation between PPGCs and mPGCs that was distinct from the pattern at E13.5. Although further supporting evidence is required, our data suggest that sex differences in histone marks exist and change throughout the course of GC development.

Histone modification was dynamically altered in $\mathrm{P} 1 \mathrm{o}$ and $\mathrm{P} 1 \mathrm{~s}$ along with germ cell differentiation with global changes in gene expression. Enrichment of $\mathrm{H} 3 \mathrm{~K} 9 \mathrm{me} 2$ alone without $\mathrm{H} 3 \mathrm{~K} 9 \mathrm{me} 3$ was found to be correlated with CGI methylation in P1s but not P1o, suggesting a cooperative role for the two types of histone modification in repressing gene expression. However, the factors regulating changes in histone marks are unknown; these may include other epigenetic mechanisms such as the chromosomal 3D conformation. The activities of histone H3K4 demethylase [42] and histone deacetylase 1/2 [43] are also important for maternal methylation imprinting and the maintenance of genomic integrity. In addition, ZFP568 was enriched at the Igf 2 promoter region, resulting in Igf2 repression without changes in DNA methylation. Interestingly, ZFP568 enrichment led to H3K9me3 accumulation in this region, suggesting a cooperative effect of modifiers and histone marks [44].

The most plausible mechanism for genome-wide epigenetic gene regulation is the concerted action of DNA methylation and other types of histone modification [14,45]. It is possible that histone marks predispose certain sites for DNA methylation in the oocyte and sperm genome, although supporting evidence for this hypothesis is needed. Although several report support this idea [20,41,46], however further research in this area is needed. In embryonic stem (ES) cells derived from mice lacking DNA methylation, genome-wide DNA demethylation altered the occupancy of histone modifications at promoters and enhancers, implying that DNA methylation is essential for the deposition of specific histone modifications across regulatory regions that coordinately influence gene expression in ES cells [47]. Whether an abundance of DNA methylation results in a similar histone modification status in ES cells and FGCs is an open question. The results in this study suggest a close relationship between DNA methylation and histone marks. It is conceivable that specific histone marks in FGCs determine the accessibility of chromatin to methyltransferases and related factors for later developmental stages.

Using a ChIP-seq protocol optimized for low cell numbers and new techniques for isolating primary and growing oocytes, we generated profiles of histone modifications associated with enhanced or reduced DNA methylation [20]. CGIs targeted for DNA methylation showed a reduced abundance of inhibitory H3K4me2 and H3K4me3 marks in both primary and growing oocytes, whereas the permissive H3K36me3 mark was enriched specifically at these CGIs in growing oocytes [20].

Here, we demonstrated that histone accumulation patterns at CGIs and their shore regions $( \pm 5 \mathrm{~kb})$ were distinguished by whether the CGIs were methylated in oocytes or sperm, or both (Figure 4). Such overview analysis did not show obvious difference between fFGCs and mFGCs, therefore further detail comparison analysis must be required to identify the latent sex specific differences. In deed, at certain gene regions clear sex specific histone modifications were identified, which strictly regulate gene expression (Figure 2D). This evidence indicates that the presence of a cluster of sex specific genes undergoing control by histone modifications in FGCs. Furthermore, the repressive marks $\mathrm{H} 3 \mathrm{~K} 27 \mathrm{me} 3, \mathrm{H} 3 \mathrm{~K} 9 \mathrm{me} 2$ and $\mathrm{H} 3 \mathrm{~K} 9 \mathrm{me} 3$ were excluded from CGIs but enriched in the shore regions (Figure 4). These results suggest a close relationship between DNA methylation and histone marks. It is conceivable that specific histone marks in FGCs determine the accessibility of chromatin to methyltransferases and related factors for later developmental stages.

ICRs - which are sites of DNA methylation imprints in both female and male germlines - exhibit differences in histone marks especially in P1o and P1s. The presence of H3K4me1 may distinguish the maternal allele, since ICRs in P1o are enriched in activating histone marks. H3K9me2 and $\mathrm{H} 3 \mathrm{~K} 9 \mathrm{me} 3$ accumulation varies in paternally methylated ICRs in P1s. Although we did not observe any histone modifications in methylated ICRs of FGCs at E13.5, imprinted methylation at ICRs is known to be established during oocyte growth in females and prospermatogonia in males [48,49]. Interestingly, five maternally imprinted genes, Gab1, Phf17, Sfmbt2, Slc38a4 and Smoc1, that are not regulated through DNA methylation but is dependent on H3K27me3 modification has recently been identified [50]; analyses at later developmental stages could provide more insight into the functional significance of this observation. It has been suggested that the reprogramming of FGCs histone modifications between E13.5 and E15.5 establishes the methylation imprint [51].

\section{Conclusion}

Our findings suggest that histone modifications play a critical role in regulating gene expression in FGCs following DNA methylation erasure, leading to their differentiation into fFGCs and mFGCs. These findings provide a novel insight into the establishment of sex-specific epigenetic modifications in the germline, which is required for 
functional germ cell development. However, further study is needed to elucidate whether and how the various types of modifications interact.

\section{Future perspective}

Illustrating the whole process of sex specification of FGCs by epigenetic mechanisms is a fascinating topic. Further detailed and comprehensive epigenetic information should be provided using a reliable and high-quality low-input ChIP-seq. Parental allele specific analysis of histone marks also contributes for better understanding the role of histone modifications. Furthermore, it is also important to gain better understanding that the overall relationship of the histone marks and other epigenetic modifications as well as genome structure. Future research should focus on understanding the direct causal relationship of histone marks with gene expressions; thus artificial alternations of histone modification states in cell level are critical for this purpose.

\section{Summary points}

- Fetal germ cells (FGCs) undergo global epigenetic reprogramming - including exchange of histone variants, remodeling of histone modifications and erasure of DNA methylation - during migration and proliferation.

- We conducted a comprehensive investigation of histone modifications in female and male FGC genomes, obtained by ChIP-seq analysis and compared these findings with datasets on DNA methylation, ATAC-seq and the overall transcriptome.

- Patterns of trimethylation of histone $\mathrm{H3}$ at lysine 4 (H3K4me3), acetylation of histone $\mathrm{H} 3$ at lysine 27 (H3K27ac) and trimethylation of histone $\mathrm{H} 3$ at lysine 27 (H3K27me3) differed between female and male FGCs.

- H3K4me3/H3K27me3 bivalent marks were enriched in different chromosomal regions of female and male FGCs, while P1 spermatogonia (P1s), which have a male-specific DNA methylation pattern, exhibited distinct histone modifications.

- The majority of genes and associated regions in both fFGCs and mFGCs were highly modified with H3K27me3, while H3K9me2 and H3K9me3 marks, which are more broadly distributed throughout the genome, were sparse.

- An assay for transposase-accessible chromatin by sequencing analysis showed that transposase-accessible chromatin regions were correlated with H3K4me3 and H3K27ac in FGCs.

- CpG islands (CGIs) - which are eventually hypermethylated in sperm - lacked H3K4me3 and H3K4me1 marks and transposase-accessible sites in FGCs.

- CGls of P1s showed distinct histone modification patterns relative to other cell types; furthermore, although there were differences in histone marks at imprinting control regions in oocytes and spermatogonia, this did not apply to FGCs.

- Our findings suggest that histone modifications play a critical role in regulating gene expression in FGCs following DNA methylation erasure, leading to their differentiation into fFGCs and mFGCs.

Supplementary data

To view the supplementary data that accompany this paper please visit the journal website at: www.futuremedicine.com/doi/suppl/10.2217/epi-2018-0193

Author contributions

T Kono, H Kobayashi and Y Matsui conceived and designed the experiments. Y Kawabata, A Kamio, Y Jincho and A Sakashita performed analysis. T Takashima performed PGC and GC collection by FACS. Y Kawabata and A Kamio performed NGS data collection and data analysis. T Kono, Y Kawabata and $\mathrm{H}$ Kobayashi wrote the paper. All authors discussed the results and commented on the manuscript.

Acknowledgments

The authors thank MC Lorincz and K Miyamoto for helpful comments on ChIP-seq and ATAC-seq, and R Ohtake for assistance with NGS data collection.

Financial \& competing interest disclosure

This project was supported by Grants-in-Aid for Scientific Research from the Japanese Science and Technology Agency and by AMED-CREST, \#JP17gm0510017h from the Japanese Agency for Medical Research and Development and MEXT-Supported Program for the Strategic Research Foundation at Private Universities, 2013-2017 (S1311017) from the Ministry of Education, Culture, 
Sports, Science and Technology of Japan. The authors have no other relevant affiliations or financial involvement with any organization or entry with a financial interest conflict with the subject matter or materials discussed in the manuscript apart from those disclosed.

No writing assistance was utilized in the production of this manuscript.

\section{Open access}

This work is licensed under the Attribution-NonCommercial-NoDerivatives 4.0 Unported License. To view a copy of this license, visit http://creativecommons.org/licenses/by-nc-nd/4.0/

\section{References}

Papers of special note have been highlighted as: • of interest; $\bullet \bullet$ of considerable interest

1 Saitou M, Kagiwada S, Kurimoto K. Epigenetic reprogramming in mouse pre-implantation development and primordial germ cells. Development 139(1), 15-31 (2012).

2 Matsui Y, Mochizuki K. A current view of the epigenome in mouse primordial germ cells. Mol. Reprod. Dev. 81(2), 160-170 (2014).

3 Morrish BC, Sinclair AH. Vertebrate sex determination: many means to an end. Reproduction 124(4), $447-457$ (2002).

4 Adams IR, Mclaren A. Sexually dimorphic development of mouse primordial germ cells: switching from oogenesis to spermatogenesis. Development 129(5), 1155-1164 (2002).

5 Seki Y, Hayashi K, Itoh K, Mizugaki M, Saitou M, Matsui Y. Extensive and orderly reprogramming of genome-wide chromatin modifications associated with specification and early development of germ cells in mice. Dev. Biol. 278(2), 440-458 (2005).

6 Hajkova P, Ancelin K, Waldmann T et al. Chromatin dynamics during epigenetic reprogramming in the mouse germ line. Nature 452(7189), 877-881 (2008).

7 Popp C, Dean W, Feng S et al. Genome-wide erasure of DNA methylation in mouse primordial germ cells is affected by AID deficiency. Nature 463(7284), 1101-1105 (2010).

8 Guibert S, Forne T, Weber M. Global profiling of DNA methylation erasure in mouse primordial germ cells. Genome Res. 22(4), 633-641 (2012).

9 Kobayashi H, Sakurai T, Miura F et al. High-resolution DNA methylome analysis of primordial germ cells identifies gender-specific reprogramming in mice. Genome Res. 23(4), 616-627 (2013).

- Povides single resolution and complete DNA methylome maps in mouse primordial germ cells at E10.5, E13.5 and E16.5 and revealed sex sepecific dynamic reprogramming of DNA methylome.

10 Kobayashi H, Sakurai T, Imai M et al. Contribution of intragenic DNA methylation in mouse gametic DNA methylomes to establish oocyte-specific heritable marks. PLoS Genet. 8(1), e1002440 (2012).

11 Shirane K, Toh H, Kobayashi H et al. Mouse oocyte methylomes at base resolution reveal genome-wide accumulation of non-CpG methylation and role of DNA methyltransferases. PLoS Genet. 9(4), e1003439 (2013).

12 Kubo N, Toh H, Shirane $\mathrm{K}$ et al. DNA methylation and gene expression dynamics during spermatogonial stem cell differentiation in the early postnatal mouse testis. BMC Genomics 16, 624 (2015).

13 Sakashita A, Kawabata Y, Jincho Y et al. Sex specification and heterogeneity of primordial germ cells in mice. PLoS ONE 10(12), e0144836 (2015).

- Provides a subset of differentially expressed genes between male and female FGCs at E13.5 using single cell and balk trasncriptome analyses.

14 Kooistra SM, Helin K. Molecular mechanisms and potential functions of histone demethylases. Nat. Rev. Mol. Cell Biol. 13(5), 297-311 (2012).

15 Azuara V, Perry P, Sauer S et al. Chromatin signatures of pluripotent cell lines. Nat. Cell. Biol. 8(5), 532-538 (2006).

16 Bernstein BE, Mikkelsen TS, Xie X et al. A bivalent chromatin structure marks key developmental genes in embryonic stem cells. Cell 125(2), 315-326 (2006).

17 Sachs M, Onodera C, Blaschke K, Ebata KT, Song JS, Ramalho-Santos M. Bivalent chromatin marks developmental regulatory genes in the mouse embryonic germline in vivo. Cell Rep. 3(6), 1777-1784 (2013).

18 Lesch BJ, Dokshin GA, Young RA, Mccarrey JR, Page DC. A set of genes critical to development is epigenetically poised in mouse germ cells from fetal stages through completion of meiosis. Proc. Natl Acad. Sci. USA 110(40), 16061-16066 (2013).

-• Reports that a set of developmentally critical genes was maintained in an epigenetically bivalent state from the initiation of sexual differentiation during fetal development and into postmeiotic stages.

19 Zheng H, Huang B, Zhang B et al. Resetting epigenetic memory by reprogramming of histone modifications in mammals. Mol. Cell 63(6), 1066-1079 (2016).

20 Stewart KR, Veselovska L, Kim J et al. Dynamic changes in histone modifications precede de novo DNA methylation in oocytes. Genes Dev. 29(23), 2449-2462 (2015). 
21 Brind'amour J, Liu S, Hudson M, Chen C, Karimi MM, Lorincz MC. An ultra-low-input native ChIP-seq protocol for genome-wide profiling of rare cell populations. Nat. Commun. 6, 6033 (2015).

-. Brind'amour J et al. developed an ultra-low-input micrococcal nuclease-based native ChIP and sequencing method and generated high-quality maps of covalent histone marks in embryonic stem cells. They identified sexually dimorphic H3K27me3 enrichment at specific genic promoters.

22 Rotem A, Ram O, Shoresh N et al. Single-cell ChIP-seq reveals cell subpopulations defined by chromatin state. Nat. Biotech. 33(11), 1165-1172 (2015).

23 Guo H, Hu B, Yan L et al. DNA methylation and chromatin accessibility profiling of mouse and human fetal germ cells. Cell Res. 27(2), 165-183 (2017).

24 Yoshimizu T, Sugiyama N, De Felice M et al. Germline-specific expression of the Oct-4/green fluorescent protein (GFP) transgene in mice. Dev. Growth Differ. 41(6), 675-684 (1999).

25 Sasagawa Y, Nikaido I, Hayashi T et al. Quartz-Seq: a highly reproducible and sensitive single-cell RNA sequencing method, reveals non-genetic gene-expression heterogeneity. Genome Biol. 14(4), R31 (2013).

26 Buenrostro JD, Wu B, Chang HY, Greenleaf WJ. ATAC-seq: a method for assaying chromatin accessibility genome-wide. Curr. Protoc. Mol. Biol. 109, 21.29.21-29 (2015).

27 Langmead B, Salzberg SL. Fast gapped-read alignment with Bowtie 2. Nat. Meth. 9(4), 357-359 (2012).

28 Ramirez F, Ryan DP, Gruning B et al. deepTools2: a next generation web server for deep-sequencing data analysis. Nucleic Acids Res. 44(W1), W160-W165 (2016).

29 Quinlan AR. BEDTools: the Swiss-army tool for genome feature analysis. Curr. Protoc. Bioinformatics 47 11.12.11-34 (2014).

30 Ye T, Krebs AR, Choukrallah MA et al. seqMINER: an integrated ChIP-seq data interpretation platform. Nucleic Acids Res. 39(6), e35 (2011).

31 Feng J, Liu T, Zhang Y. Using MACS to identify peaks from ChIP-Seq data. Curr. Protoc. Bioinformatics Chapter 2(Unit 2), 14 (2011).

32 Love MI, Huber W, Anders S. Moderated estimation of fold change and dispersion for RNA-seq data with DESeq2. Genome Biol. 15(12), 550 (2014).

33 Trapnell C, Roberts A, Goff L et al. Differential gene and transcript expression analysis of RNA-seq experiments with TopHat and Cufflinks. Nat. Protocol. 7(3), 562-578 (2012).

34 Ernst J, Kellis M. ChromHMM: automating chromatin-state discovery and characterization. Nat. Methods 9(3), 215-216 (2012).

35 Deaton AM, Bird A. CpG islands and the regulation of transcription. Genes Dev. 25(10), 1010-1022 (2011).

36 Boyle AP, Song L, Lee BK et al. High-resolution genome-wide in vivo footprinting of diverse transcription factors in human cells. Genome Res. 21(3), 456-464 (2011).

37 Nakaki F, Hayashi K, Ohta H, Kurimoto K, Yabuta Y, Saitou M. Induction of mouse germ-cell fate by transcription factors in vitro. Nature 501(7466), 222-226 (2013).

38 Kee K, Angeles VT, Flores M, Nguyen HN, Reijo Pera RA. Human DAZL, DAZ and BOULE genes modulate primordial germ-cell and haploid gamete formation. Nature 462(7270), 222-225 (2009).

39 Liu S, Brind'amour J, Karimi MM et al. Setdb1 is required for germline development and silencing of H3K9me3-marked endogenous retroviruses in primordial germ cells. Genes Dev. 28(18), 2041-2055 (2014).

40 Voigt P, Tee WW, Reinberg D. A double take on bivalent promoters. Genes Dev. 27(12), 1318-1338 (2013).

41 Hanna CW, Taudt A, Huang J et al. MLL2 conveys transcription-independent H3K4 trimethylation in oocytes. Nat. Struct. Mol. Biol. 25(1), 73-82 (2018).

-. Reveals H3K4me3, H3K27ac and H3K27me3 modification profiles throughout mouse oogenesis.

42 Ciccone DN, Su H, Hevi S et al. KDM1B is a histone H3K4 demethylase required to establish maternal genomic imprints. Nature 461(7262), 415-418 (2009).

43 Ma P, De Waal E, Weaver JR, Bartolomei MS, Schultz RM. A DNMT3A2-HDAC2 Complex Is Essential for Genomic Imprinting and Genome Integrity in Mouse Oocytes. Cell. Rep. 13(8), 1552-1560 (2015).

44 Yang P, Wang Y, Hoang D et al. A placental growth factor is silenced in mouse embryos by the zinc finger protein ZFP568. Science 356(6339), 757-759 (2017).

45 Feil R, Fraga MF. Epigenetics and the environment: emerging patterns and implications. Nat. Rev. Genet. 13(2), 97-109 (2012).

46 Ciccone DN, Chen T. Histone lysine methylation in genomic imprinting. Epigenetics 4(4), 216-220 (2009).

47 King AD, Huang K, Rubbi L et al. Reversible regulation of promoter and enhancer histone landscape by DNA methylation in mouse embryonic stem cells. Cell. Rep. 17(1), 289-302 (2016).

48 Hiura H, Obata Y, Komiyama J, Shirai M, Kono T. Oocyte growth-dependent progression of maternal imprinting in mice. Genes Cells 11(4), 353-361 (2006). 
49 Hiura H, Komiyama J, Shirai M, Obata Y, Ogawa H, Kono T. DNA methylation imprints on the IG-DMR of the Dlk1-Gtl2 domain in mouse male germline. FEBS Lett. 581(7), 1255-1260 (2007).

50 Inoue A, Jiang L, Lu F, Suzuki T, Zhang Y. Maternal H3K27me3 controls DNA methylation-independent imprinting. Nature 547(7664), 419-424 (2017).

51 Henckel A, Chebli K, Kota SK, Arnaud P, Feil R. Transcription and histone methylation changes correlate with imprint acquisition in male germ cells. EMBO J. 31(3), 606-615 (2012).

52 Robinson JT, Thorvaldsdottir H, Winckler W et al. Integrative genomics viewer. Nat. Biotech. 29(1), 24-26 (2011).

53 Mclean CY, Bristor D, Hiller M et al. GREAT improves functional interpretation of cis-regulatory regions. Nat. Biotech. 28(5), 495-501 (2010).

54 Huang Da W, Sherman BT, Lempicki RA. Systematic and integrative analysis of large gene lists using DAVID bioinformatics resources. Nat. Protocol. 4(1), 44-57 (2009). 
